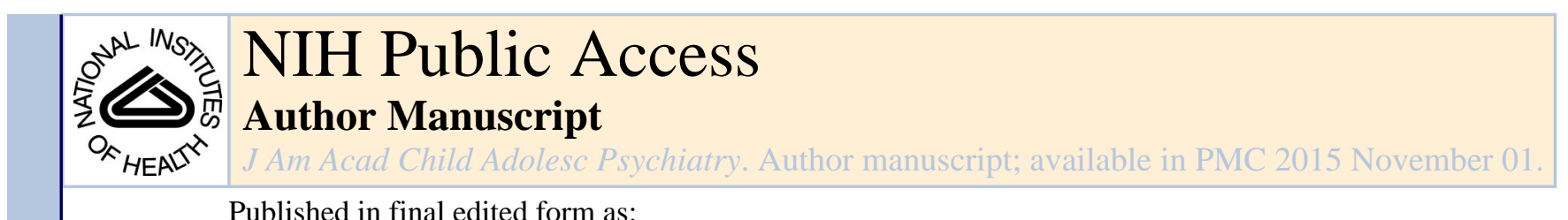

Published in final edited form as:

J Am Acad Child Adolesc Psychiatry. 2014 November ; 53(11): 1216-1224. doi:10.1016/j.jaac.

2014.08.004.

\title{
Repetitive Behavior in 12-Month-Olds Later Classified With Autism Spectrum Disorder
}

\begin{abstract}
Jed T. Elison, PhD, Jason J. Wolff, PhD, J. Steven Reznick, PhD, Kelly N. Botteron, MD, Annette M. Estes, PhD, Hongbin Gu, PhD, Heather C. Hazlett, PhD, Adriane J. Meadows, BA, Sarah J. Paterson, PhD, Lonnie Zwaigenbaum, MD, and Joseph Piven, MD for the IBIS Network

Dr. Elison is with the Institute of Child Development at the University of Minnesota, Minneapolis.

Drs. Wolff, Gu, Hazlett, Piven, and Ms. Meadows are with the Carolina Institute for Developmental Disabilities and the University of North Carolina (UNC) at Chapel Hill, NC. Dr. Reznick is also with the University of North Carolina at Chapel Hill. Dr. Botteron is with Washington University in St. Louis. Dr. Estes is with the University of Washington, Seattle. Dr. Paterson is with the Children's Hospital of Philadelphia. Dr. Zwaigenbaum is with the University of Alberta, Edmonton, Alberta, Canada.
\end{abstract}

\section{Abstract}

Objective-As compared to the utility of early emerging social communicative risk markers for predicting a later diagnosis of autism spectrum disorder (ASD), less is known about the relevance of early patterns of restricted and repetitive behaviors. We examined patterns of stereotyped motor mannerisms and repetitive manipulation of objects in 12-month-olds at high and low risk for developing ASD, all of whom were assessed for ASD at 24 months.

Method-Observational coding of repetitive object manipulation and stereotyped motor behaviors in digital recordings of the Communication and Symbolic Behavior Scales was conducted using the Repetitive and Stereotyped Movement Scales for three groups of 12-montholds: 1) low-risk infants ( $\mathrm{LR}, \mathrm{n}=53$ ); 2) high-familial-risk infants who did not meet diagnostic criteria for ASD at 24-months (HR-negative, $n=75$ ); and 3) high-familial-risk infants who met diagnostic criteria for ASD at 24 months (HR-ASD, $\mathrm{n}=30$ ).

() 2014 American Academy of Child and Adolescent Psychiatry. All rights reserved.

Correspondence to Jed T. Elison, Institute of Child Development, University of Minnesota, 51 East River Parkway, Minneapolis, MN 55455; jtelison@umn.edu.

The Infant Brain Imaging Study (IBIS) Network is a National Institutes of Health (NIH)-funded Autism Center of Excellence project and consists of a consortium of 7 universities in the US and Canada. Clinical Sites: University of North Carolina: J. Piven, MD (IBIS Network PI), H.C. Hazlett, PhD, J.C. Chappell, MS; University of Washington: S. Dager, MD, A. Estes, PhD, D. Shaw, MD; Washington University: K. Botteron, MD, R. McKinstry, MD, PhD, J. Constantino, MD, J. Pruett, MD, PhD; Children's Hospital of Philadelphia: R. Schultz, PhD, S. Paterson, PhD; University of Alberta: L. Zwaigenbaum, MD; Data Coordinating Center, Montreal Neurological Institute: A.C. Evans, PhD, D.L. Collins, PhD, G.B. Pike, PhD, P. Kostopolous, PhD, S. Das, BSc; Image Processing Core, University of Utah: G. Gerig, PhD; University of North Carolina: M. Styner, PhD; Statistical Analysis Core, University of North Carolina: H. Gu, PhD; Genetics Analysis Core, University of North Carolina: P. Sullivan, MD, F. Wright, PhD.

Publisher's Disclaimer: This is a PDF .le of an unedited manuscript that has been accepted for publication. As a service to our customers we are providing this early version of the manuscript. The manuscript will undergo copyediting, typesetting, and review of the resulting proof before it is published in its final citable form. Please note that during the production process errors may be discovered which could affect the content, and all legal disclaimers that apply to the journal pertain.

Dr. Estes and Ms. Meadows report no biomedical financial interests or potential conflicts of interest. 
Results-The HR-ASD group showed significantly more stereotyped motor mannerisms than both the HR-negative group ( $p=.025)$ and the LR group $(p=.001)$. The HR-ASD and HRnegative groups demonstrated statistically equivalent repetitive object manipulation scores $(p=$. 431 ), and both groups showed significantly more repetitive object manipulation than the LR group ( $p$ 's < 0.040). Combining the motor and object stereotypy scores into an RSMS composite yielded a disorder-continuum effect such that each group was significantly different from one another (LR $<$ HR-negative < HR-ASD).

Conclusion-These results suggest that targeted assessment of repetitive behavior during infancy may augment early identification efforts.

\section{Keywords}

autism; repetitive behavior; motor stereotypies; infant siblings; development

\section{Introduction}

Accumulating evidence suggests that a number of social communicative risk markers observed as early as 12 months of age distinguish infants who will later meet diagnostic criteria for autism spectrum disorder (ASD) ${ }^{1-5}$. Comparatively less is known about whether restricted and repetitive behaviors (RRBs) represent similar levels of risk for a later diagnosis. Comprehensive characterization of restricted and repetitive behavior at 12 months has important diagnostic implications ${ }^{6}$ and is also relevant for developmental models of pathogenesis.

In a cohort of 18- to 24-month-old toddlers, ascertained via a general population screening of healthcare and childcare agencies, Wetherby and colleagues ${ }^{7-9}$ observed that repetitive object manipulation and stereotyped motor behaviors differentiate toddlers with ASD from typically developing toddlers and toddlers with developmental delays. These data complement and extend evidence based on parent-report and observational diagnostic tools that highlight the importance of repetitive behaviors for distinguishing toddlers and preschool-aged children with ASD from comparison groups ${ }^{10-14}$. Lower-order repetitive behaviors ${ }^{15}$ observed in clinically ascertained samples and repetitive use of objects in particular represent early emerging and persistent features of ASD present from 2 to 9 years of age ${ }^{14}$, and the severity of these behaviors at 2 years of age predicts prognosis seven years later ${ }^{16}$.

Comparing children with developmental delays to children with ASD has elucidated aspects of RRBs that are specific to the ASD phenotype. However, additional comparison groups are needed to determine whether detailed characterization of RRBs might inform the underlying biological architecture of ASD. For example, Wolff and colleagues ${ }^{17}$ observed differences in repetitive behavior profiles between preschool-aged boys with idiopathic autism and boys with fragile $\mathrm{X}$ syndrome who also met diagnostic criteria for ASD. Evidence from family studies of affected sibling pairs suggests that higher-order repetitive behaviors tend to aggregate in families ${ }^{18-19}$. Further evidence from a dense, extended pedigree study suggests unique linkage signals for RRBs ${ }^{20}$. Another family study examining a dimensional index of motor functioning found striking similarities in the degree of motor 
impairment among affected sibling pairs, $83 \%$ of whom had scores of at least one standard deviation below the general population mean ${ }^{21}$. However, only $6 \%$ of unaffected siblings of probands with ASD performed below one standard deviation of the population mean.

Several prospective studies of infants at high familial risk for developing ASD have examined RRBs. In these studies, high risk is defined as having an older sibling diagnosed with ASD, and low risk is defined as having a typically developing older sibling and no first- or second-degree relatives with autism. Results from a micro-behavioral coding analysis in which four objects were briefly presented to high- and low-risk infants suggested that those who later developed ASD $(n=9)$ showed specific patterns of unusual visual and manual exploration of objects at 12 months of age ${ }^{22}$. The comparison groups in this study included an 'other delays' group $(\mathrm{n}=10)$ and a 'no concerns' group $(\mathrm{n}=47)$. In another study, high-risk 18-month-olds later diagnosed with ASD ( $\mathrm{n}=17)$, high-risk siblings who showed other delays $(\mathrm{n}=12)$, and high-risk siblings who showed no developmental delays $(\mathrm{n}=19)$ demonstrated more nonfunctional repetitive play acts with objects than low-risk toddlers ${ }^{23}$. Although comparisons were not conducted between the high-risk subgroups, this analysis suggests that nonfunctional repetitive play may be a familial marker of ASD. Atypical motor mannerisms (e.g., arm waving) have differentiated 18-month-olds who later developed ASD $(\mathrm{n}=8)$ from low-risk infants and high-risk infants who did not develop ASD; however, this pattern was not observed at 12 months of age ${ }^{24}$. An additional preliminary study examining the rate and inventory of object and motor stereotypies in 15-month-olds points to the possibility that high-risk infants who do not develop ASD $(n=12)$ show higher rates of these behaviors than low-risk infants ${ }^{25}$. In sum, findings to date suggest that some features of repetitive behavior may emerge as early as 12 or 18 months of age in children who develop ASD. However, small sample sizes, investigations of isolated features (i.e., either repetitive object manipulation or stereotyped motor behaviors), and decisions to merge highrisk and low-risk groups have limited the impact of findings to date.

In the current study, we employed a standardized behavioral coding scheme ${ }^{7}$ to examine patterns of motor stereotypies and repetitive object manipulation across 3 groups of 12month-olds that maintains the integrity of the family design: 1) high-risk infants later diagnosed with ASD; 2) high-risk infants who do not meet diagnostic criteria for ASD; and 3) low-risk infants. The primary objective was to characterize patterns of RRBs representative of either a disorder-specific deficit or a pattern representing familial liability for ASD in a large sample of 12-month-olds $(\mathrm{N}=159)$.

\section{Method}

This study took place in the context of an ongoing Autism Center of Excellence Network study (the Infant Brain Imaging Study; IBIS) prospectively investigating longitudinal brain and behavioral trajectories in high- and low-risk infants. The institutional review boards at all sites approved the research protocol, and parents provided informed consent for their infants to participate. For additional information on the study design, see Elison et al. $(2013)^{26}$ and Wolff et al. (2012) ${ }^{27}$. 


\section{Participants}

All infants were assessed at one of four clinical sites: the University of North Carolina at Chapel Hill, the University of Washington, the Children's Hospital of Philadelphia, and Washington University in St. Louis. The study sample included children with a digital video recording of the Communication and Symbolic Behavior Scales-Developmental Profile (CSBS-DP) ${ }^{28}$ collected at the 12-month visit, and clinical outcome data at the 24-month visit. Exclusion criteria for both high-risk and low-risk infants included the following: 1) history of known genetic conditions or syndromes associated with ASD;2) significant medical conditions affecting growth, development or cognition or sensory impairments such as significant vision or hearing loss; 3) birth weight $<2,000$ grams and/or gestational age < 36 weeks; 4) history of significant perinatal adversity, or exposure in-utero to neurotoxins; 5) contraindication for magnetic resonance imaging (MRI); 6) predominant home language other than English; 7) having been adopted; and 8) family history of a first-degree relative with psychosis, schizophrenia, or bipolar disorder. Low-risk infants were excluded for a family history of a first- or second-degree relative with autism, intellectual disability, or older sibling (proband) with ASD symptoms measured with the Social Communication Questionnaire ${ }^{29}$. Clinical diagnosis of ASD was corroborated in the probands of high-risk infants with the Autism Diagnostic Interview - Revised ${ }^{30}$. The present study included 158 infants who were classified into three outcome groups: low-risk (hereafter LR, $n=53$ ), highrisk ASD-negative (hereafter HR-neg, $n=75$ ), and high-risk ASD-positive (hereafter HRASD, $n=30$ ), based on risk status and diagnostic outcome. To be included in the HR-ASD group, children met cut-off criteria for autism or ASD on the Autism Diagnostic Observation Schedule (ADOS), ${ }^{31}$ and diagnostic criteria for autism according to DSM-IV criteria as determined by clinical-best-estimate made by experienced, licensed clinicians. One LR infant met criteria for an ASD and was excluded from the current study to maintain the structure of the familial-high-risk design. See Table 1 for sample characteristics.

\section{Experimental Procedure}

The behavioral sample of the CSBS-DP ${ }^{27}$ is a standardized procedure designed to elicit social and communicative behaviors in infants between 12 and 24 months of age. The interaction between examiner and infant is divided into 6 sampling opportunities: 1) windup toy; 2) balloon; 3) bubbles; 4) jar; 5) books; and 6) play, generally lasting 15-30 minutes. The interaction during the CSBS-DP Behavioral Sample was digitally recorded (video) for subsequent coding with the RSMS (see below).

The Repetitive and Stereotyped Movement Scales (RSMS) ${ }^{7}$ is a clinical coding scheme designed as a companion to the CSBS Behavioral Sample. Directly informed by previous findings ${ }^{8-9,32}$, the RSMS was developed to assess the rate and inventory of stereotyped motor behaviors and repetitive object manipulation in real time. The stereotyped motor behaviors coded in the RSMS include 1) flapping arms and hands; 2) pats, taps, or presses body part; 3) rubs body part; and 4) stiffens fingers, hands, or arms. The behaviors captured under the repetitive object manipulation category include 1) swipes object;2) rubs or squeezes object; 3) rolls or knocks over object; 4) rocks, flips, turns over, or flicks object; 5) spins or wobbles object; 6) collects objects; 7) moves or places objects to one location; 8) lines up or stacks objects; and 9) clutches object. Many of the coding parameters were 
derived from seminal work by Thelen ${ }^{33}$. The coding scheme yields two subdomain scores (body and object clusters) and a total RSM composite score.

All coding was conducted blind to risk and outcome status. The first author coded each digital file, and $25 \%$ of files were randomly selected for coding by a second rater to assess reliability. Intraclass correlation coefficients (ICC) indicated excellent inter-rater reliability for the body cluster (ICC $=0.90$ ), the object cluster $(\mathrm{ICC}=0.93$ ), and the RSM composite $(\mathrm{ICC}=0.92)$.

\section{Cognitive and Clinical Assessment}

The Mullen Scales of Early Learning (MSEL) ${ }^{34}$ is a standardized measure of cognitive and motor development for children from birth to 68 months that assesses skills and abilities in five domains: gross motor, visual reception, fine motor, receptive language, and expressive language. This measure yields a composite standard score (Early Learning Composite or ELC), reflecting overall cognitive ability as well as subdomain $\mathrm{T}$ and age-equivalent scores. Following precedent ${ }^{32}$, a nonverbal developmental quotient (NVDQ) and verbal developmental quotient (VDQ) were derived from the raw MSEL data. The NVDQ was derived from the average age equivalent scores from the fine motor and visual reception domains divided by the chronological age at assessment multiplied by 100 (i.e., mental age/ chronological age $\times 100$ ). A similar formula was used to derive the VDQ from the receptive and expressive language subscales.

The $\operatorname{ADOS}^{30}$ is a semi-structured assessment of communication, social interaction, play skills, and restricted and repetitive behavior administered by trained examiners to all participants at the 24-month visit. The ADOS was conducted and scored by experienced, research-reliable examiners. The ADOS shows better sensitivity than specificity when used with young children ${ }^{35}$; therefore we expected some children to exceed ASD threshold on the ADOS but not receive a clinical-best-estimate diagnosis.

\section{Analytic Strategy}

We performed a Multivariate Analysis of Covariance (MANCOVA) to test for differences between groups while controlling for cognitive level (i.e., Mullen ELC) and simultaneously accounting for covariance between dependent measures. The omnibus test statistic was decomposed with univariate analyses (Type III sum of squares), and planned pairwise comparisons were reported for significant group differences per each dependent variable. We then explored the associations between the three primary RSMS variables with cognitive and clinical characteristics among the groups, as determined by the pattern of results in planned pairwise comparisons.

\section{Results}

No statistically significant differences were observed between the three groups in chronological age at the 12-month assessment $(p=.709)$, weeks gestation $(p=.296)$, race/ ethnicity $(p=.140)$, maternal education level $(p=.200)$, family income $(p=.653)$, or chronological age at the 24-month visit $(p=.413)$. The sex ratio of the groups differed significantly $(p=.036)$, with a higher proportion of males in the ASD group, as would be 
expected. A MANCOVA with the body cluster subscale, object cluster subscale, and RSMS composite scores included as dependent variables, group status included as a fixed factor, and the ELC from the Mullen entered as a covariate revealed a significant main effect of group status (Pillai's Trace $V=0.101, F_{(3,153)}=2.708, p=.014, \eta_{p}{ }^{2}=0.050$ ) and no effect of ELC $(p=.320)$. Univariate ANOVAs confirmed the unique effects of group status on the body cluster $\left(F_{(2,154)}=5.614, p=.004, \eta_{p}{ }^{2}=0.068\right)$, the object cluster $\left(F_{(2,154)}=3.141, p\right.$ $\left.=.046, \eta_{p}{ }^{2}=0.039\right)$, and the RSMS composite score $\left(F_{(2,154)}=6.935, p=.001, \eta_{p}{ }^{2}=\right.$ $0.083)$.

Planned pairwise comparisons revealed that the three groups significantly differed from one another on the RSMS composite index. The HR-ASD group showed significantly higher scores than both the HR-neg $(p=.046)$ and the LR $(p<.001)$ groups. Furthermore, the HRneg group significantly differed from the LR group $(p=.015)$. Considering the object cluster score, the LR group scored significantly lower than the HR-ASD group $(p=.027)$ and the HR-neg group $(p=.040)$. The two HR groups showed statistically equivalent object cluster scores $(p=.431)$. Finally, the HR-ASD group significantly differed from both the HR-neg ( $p$ $=.025)$ and the LR $(p=.001)$ groups on the body cluster index. The HR-neg and LR groups were not statistically different on the body cluster score $(p=.082)$. See Figure 1 and Table 2 for additional information.

\section{Exploratory Analyses: Associations between the RSMS and Developmental Level}

We explored associations between RSMS subscales and developmental level (i.e., NVDQ, VDQ, gross motor $t$ score) according to the patterns of group differences that emerged from the planned comparisons above. As the HR-ASD group differed from both the LR and HRneg group on the body cluster index, separate correlation coefficients were examined for the HR-ASD and the combined LR+HR-neg groups. Within the HR-ASD group $(n=30)$, standardized scores on the gross motor subscale of the MSEL were significantly associated with the body cluster score $\left(r_{s}=-0.465, p=.010\right)$. Within the combined LR and HR-neg group $(\mathrm{n}=128)$, the body cluster score was significantly associated with VDQ $\left(r_{s}=-0.198, p\right.$ $=.025)$.

Because the LR group differed from the HR-neg and HR-ASD groups on the object cluster index, separate correlation coefficients were examined for the LR and the combined HR-neg + HR-ASD groups in relation to this variable. We observed no significant associations between the object cluster scores and developmental level in the LR group. In the combined HR-neg and HR-ASD group ( $\mathrm{n}=105)$, we observed a significant association, albeit small, between the object cluster score and VDQ $\left(r_{s}=-0.194, p=.048\right)$.

Considering the above associations with VDQ among the distinct subgroups, we examined the association between VDQ and RSMS subscales in the entire group $(\mathrm{N}=158)$. VDQ was associated with the body cluster $\left(r_{s}=-0.186, p=.019\right)$, the object cluster $\left(r_{s}=-0.257, p=\right.$. $001)$, and the RSMS composite score $\left(r_{s}=-0.305, p<.001\right)$. The alpha value for the correlational analyses was not corrected for multiple comparisons, as these were deemed to be exploratory, hypothesis-generating analyses. 


\section{Discussion}

We used a standardized behavioral coding scheme to characterize repetitive object and motor stereotypies in infants at low- and high-familial risk for developing ASD, a subset of whom met diagnostic criteria for ASD at 24 months of age. RSMS composite scores significantly differentiated the three groups from one another (LR $<$ HR-neg $<$ HR-ASD). Decomposing this finding revealed that the HR-ASD and the HR-neg groups did not differ on the object cluster subscale, but that both groups differed from the LR group (LR < HRneg $=$ HR-ASD), suggesting that repetitive object manipulation at 12 months relates primarily to genetic liability for ASD rather than representing a specific precursor to the development of ASD. However, the body cluster subscale yielded a pattern of results suggesting a disorder specific deficit (LR $=$ HR-neg $<$ HR-ASD). Accumulating evidence suggests that the restricted and repetitive behavior domain does not represent a unitary construct $^{15,17-19}$, and the current findings suggest that this empirical observation may hold for 12-month-olds.

The current findings suggest that clinical characterization of atypical motor stereotypies may augment early identification of ASD in 12-month-olds, a finding that supplements recent evidence that parent report of restricted and repetitive behavior at 12 months differentiates infants who will later develop $\mathrm{ASD}^{36}$. The body cluster score is derived from 1) the rate of stereotyped motor mannerisms and 2) the inventory or variety of stereotyped motor mannerisms observed within the given sampling period. Examining these components of the body cluster index as summarized in Table 2 (i.e., variety/inventory of the behaviors observed and the rate at which they were observed) suggests that the rate of stereotyped motor behaviors distinguishes the HR-ASD group from the LR and the HR-neg groups in the same manner as the overall body cluster index. However, the variety or inventory of repetitive motor stereotypies did not significantly differ between the HR-ASD and the HRneg groups. Thelen ${ }^{33}$ reported that the rate of motor stereotypies significantly decreases between 8 and 12 months of age in typically developing infants, but that the inventory or number of different stereotypies remains statistically equivalent during this period. Twelvemonth-old infants later diagnosed with ASD may not proceed through a developmental reduction in the rate of motor stereotypies during the typical timeframe, thus exhibiting rates of behaviors equivalent to that of typically developing infants between 6 and 10 months of age. A similar phenomenon, characterized by an atypical sequence of repetitive motor behavior that is both later to arrive and longer to resolve relative to typically developing peers, has been recorded among young children with either Down syndrome or motor impairment ${ }^{37}$. An alternative explanation would be warranted if infants later classified with ASD exhibited significantly higher rates of motor stereotypies compared to LR and HR-neg infants throughout the first year of life. More frequent, longitudinal assessment across a broader age range is required to test this hypothesis.

In the HR-ASD infants, the body cluster score was associated with individual differences in gross motor ability at 12 months such that higher gross motor scores were associated with lower body cluster scores. We did not observe group differences in gross motor ability at 12 months in this sample; however, gross motor development in the latter half of the first year of life may be related to the atypical rate of stereotyped motor mannerisms observed in the 
current study. Additionally, the observation of a small but significant correlation between verbal developmental level and the body cluster score in infants who do not develop autism may contribute to enduring efforts to understand the dynamic association between vocalmotor and speech-gesture system development during infancy ${ }^{38}$.

Repetitive object manipulation at 12 months does not appear to distinguish HR infants who later develop ASD from those who do not but rather appears to index familial liability for ASD. The current pattern of results motivates several intriguing conjectures including 1) the possibility that different underlying mechanisms may contribute to the same observable behavior (i.e., related to the concept of the phenocopy) in the HR infants as a whole; 2) the potential for shared environmental and/or genetic factors to contribute to atypical object manipulation in HR infants; and 3) the potential that diagnostic classification at 24 months, and the inclusion of a small percentage of children in the HR-negative group who will later meet diagnostic criteria for ASD, may mask true differences in repetitive object manipulation. Morgan and colleagues ${ }^{7}$ posited that object manipulation at this age might function as a developmental precursor to higher-order repetitive behaviors, such as insistence on sameness and restricted interests, whereas stereotyped motor mannerisms observed during the RSMS represent behaviors consistent with lower-order repetitive behaviors. We observed associations between verbal developmental level and both object and motor stereotypies, which suggests that a common mechanism captured by VDQ may be contributing to both domains. More research on the discriminant validity of these domains is needed to refute the hypothesis suggested by Morgan and colleagues ${ }^{7}$.

Object manipulation/exploration during infancy is thought to play an important role in cognitive development ${ }^{38-42}$. Research has demonstrated that repetitive use of objects and atypical motor mannerisms tend to load together on factor analytic studies of ASD ${ }^{13,18-19}$, and that this factor is commonly associated with cognitive level in children, adolescents, and adults $^{18,43}$. Future research is needed to determine the developmental association between object manipulation/exploration during infancy and repetitive object use in the preschool years. The factors that mediate this transition may be particularly meaningful for understanding the pathogenesis of autism and/or the protective factors for HR infants who do not develop ASD. Family studies that characterize phenomena related to developmental timing optimize the potential for discovering biological markers associated with developmentally and genetically informative alternative phenotypes not yet obfuscated by abnormal developmental trajectories. Consistent with previous findings ${ }^{23}$, the current data suggest that abnormal repetitive manipulation of objects may be a developmentally meaningful alternative phenotype ${ }^{44}$.

Several limitations of the current study warrant mention. Although diagnostic stability remains high in clinically ascertained samples of 2-year-olds ${ }^{45-46}$ and diagnosis at 2 years is a well-established benchmark in both research and clinical practice ${ }^{47-48}$, it is unknown what percentage of the infant-siblings classified as high-risk-negative at 24 months of age will actually meet diagnostic criteria for ASD at a later age. Comprehensive characterization at a later age would support a more definitive classification and potentially allow subgrouping of the HR-negative group. This group is exceptionally heterogeneous, and there is evidence that a substantial proportion of these children will not develop on a typical trajectory ${ }^{49-50}$. 
Lastly, the RSMS is constrained by the semi-structured assessment in which it is conducted and consequently may only capture a subset of possible RRBs operating during this developmental period.

When considered in conjunction with findings from parent report ${ }^{36}$ and in the context of the broader literature delineating observable social communication deficits at 12 months of age, the current data suggest that restricted and repetitive behaviors are observed as early as social communication deficits in infants who later meet diagnostic criteria for ASD. Future investigations into the underlying neural circuitry (e.g., sensory-motor pathways) associated with these atypical behaviors during the first year of life promises to elucidate mechanisms of pathogenesis of ASD.

\section{Acknowledgments}

This research was supported by grants awarded to J.P. from NIH/National Institute of Child Health and Human Development (NICHD) (R01 HD055741, HD055741-S1, P30 HD03110, T32 HD40127) Autism Speaks, and the Simons Foundation. J.T.E. was supported by NIH/NICHD grant 5-T32-HD007376, and aspects of this work contributed to his $\mathrm{PhD}$ dissertation while affiliated with UNC. J.T.E. had full access to the data and takes full responsibility for the integrity and accuracy of the data analysis.

Drs. Elison and Gu served as the statistical experts for this research.

The authors wish to thank the IBIS children and families for their ongoing participation in this longitudinal study. They also wish to thank Chad Chappell, MS, from UNC-Chapel Hill, Samir Das, BSc, and Penelope Kostopoulos, $\mathrm{PhD}$, both from the Montreal Neurological Institute, for their contributions. They also thank Amy Wetherby, PhD, and Lindee Morgan, PhD, both from Florida State University, for training J.T.E on the RSMS coding scheme.

Disclosure: Dr. Elison has received grant or research funding from the National Children's Study and the National Institute of Mental Health (NIMH). Dr. Wolff has received grant or research funding from NIMH. Dr. Reznick has received grant or research funding from the National Children's Study. Dr. Botteron has received grant or research support from NICHD, the National Institute of Biomedical Imaging and Bioengineering, NIMH, and Autism Speaks. Dr. Gu has received grant or research funding from NIMH. Dr. Hazlett has received grant or research funding from NIMH. Dr. Paterson has received grant or research funding from the National Children's Study. Dr. Zwaigenbaum is the site PI of a study sponsored by SynapDx (receives operating funds but no honoraria) and has received grant or research support from the Canadian Institutes of Health Research, NeuroDevNet, Alberta Innovates - Health Solutions, Stollery Children's Hospital Foundation, Autism Intervention Research - Physical Health. Dr. Piven has received grant or research funding from NIMH and has served as a consultant to the John Merck Foundation and to the Mindich Foundation.

\section{References}

1. Hutman T, Rozga A, DeLaurentis AD, Barnwell JM, Sugar CA, Sigman M. Response to distress in infants at risk for autism: a prospective longitudinal study. J Child Psychol Psychiatry. 2010; 51:1010-1020. [PubMed: 20546081]

2. Landa R, Holman KC, Garrett-Mayer E. Social and communication development in toddlers with early and later diagnosis of autism spectrum disorders. Arch Gen Psychiatry. 2007; 64:853-864. [PubMed: 17606819]

3. Ozonoff S, Iosif A, Baguio F, et al. A prospective study of the emergence of early behavioral signs of autism. J Am Acad Child Adolesc Psychiatry. 2010; 49:256-266. [PubMed: 20410715]

4. Rozga A, Hutman T, Young GS, et al. Behavioral profiles of affected and unaffected siblings of children with autism: contribution of measures of mother-infant interaction and nonverbal communication. J Autism Dev Disord. 2011; 41:287-301. [PubMed: 20568002]

5. Zwaigenbaum L, Bryson S, Rogers T, Roberts W, Brian J, Szatmari P. Behavioral manifestations of autism in the first year of life. Int J Dev Neurosci. 2005; 23:143-152. [PubMed: 15749241] 
6. Guthrie W, Swineford LB, Wetherby AM, Lord C. Comparison of DSM-IV and DSM-5 factor structure models for toddlers with autism spectrum disorder. J Am Acad Child Adolesc Psychiatry. 2013; 52:797-805. [PubMed: 23880490]

7. Morgan L, Wetherby AM, Barber A. Repetitive and stereotyped movements in children with autism spectrum disorders late in the second year of life. J Child Psychol Psychiatry. 2008; 49:826-837. [PubMed: 18503532]

8. Barber AB, Wetherby AM, Chambers NW. Brief report: repetitive behaviors in young children with autism spectrum disorder and developmentally similar peers: a follow up to Watt etal. (2008). J Autism Dev Disord. 2012; 42:2006-2012. [PubMed: 22222776]

9. Watt N, Wetherby AM, Barber A, Morgan L. Repetitive and stereotyped behaviors in children with autism spectrum disorders in the second year of life. J Autism Dev Disord. 2008; 38:1518-1533. [PubMed: 18266099]

10. Bishop SL, Richler J, Lord C. Association between restricted and repetitive behaviors and nonverbal IQ in children with autism spectrum disorders. Child Neuropsychol. 2006; 12:247-267. [PubMed: 16911971]

11. Kim SH, Lord C. Restricted and repetitive behaviors in toddlers and preschoolers with autism spectrum disorders based on the autism diagnostic observation scale (ADOS). Autism Res. 2010; 3:162-173. [PubMed: 20589716]

12. Mirenda P, Smith IM, Vaillancourt T, et al. Validating the repetitive behavior scale-revised in young children with autism spectrum disorder. J Autism Dev Disord. 2010; 40:1521-1530. [PubMed: 20405194]

13. Richler J, Bishop SL, Kleinke JR, Lord C. Restricted and repetitive behaviors in young children with autism spectrum disorders. J Autism Dev Disord. 2007; 37:73-85. [PubMed: 17195920]

14. Richler J, Huerta M, Bishop SL, Lord C. Developmental trajectories of restricted and repetitive behaviors and interests in children with autism spectrum disorders. Dev Psychopathol. 2010; 22:55-69. [PubMed: 20102647]

15. Turner M. Annotation: repetitive behavior in autism: a review of psychological research. J Child Psychol Psychiatry. 1999; 40:839-849. [PubMed: 10509879]

16. Lord C, Risi S, DiLavore PS, Shulman C, Thurm A, Pickles A. Autism from 2 to 9 years of age. Arch Gen Psychiatry. 2006; 63:694-701. [PubMed: 16754843]

17. Wolff JJ, Bodfish JW, Hazlett HC, Lightbody AA, Reiss AL, Piven J. Evidence of a distinct behavioral phenotype in young boys with fragile X syndrome and autism. J Am Acad Child Adolesc Psychiatry. 2012; 51:1324-1332. [PubMed: 23200289]

18. Lam KS, Bodfish JW, Piven J. Evidence for three subtypes of repetitive behavior in autism that differ in familiality and association with other symptoms. J Child Psychol Psychiatry. 2008; 49:1193-1200. [PubMed: 19017031]

19. Szatmari P, Georgiades S, Bryson S, et al. Investigating the structure of the restricted, repetitive behaviours and interests domain of autism. J Child Psychol Psychiatry. 2006; 47:582-590. [PubMed: 16712635]

20. Piven J, Vieland VJ, Parlier M, et al. A molecular genetic study of autism and related phenotypes in extended pedigrees. J Neurodev Disord. 2013; 5:30. [PubMed: 24093601]

21. Hilton CL, Zhang Y, Whilte MR, Klohr CL, Constantino J. Motor impairment in sibling pairs concordant and discordant for autism spectrum disorders. Autism. 2012; 16:430-441. [PubMed: 22013131]

22. Ozonoff S, Macari S, Young GS, Goldring S, Thompson M, Rogers SJ. Atypical object exploration at 12 months of age is associated with autism in a prospective sample. Autism. 2008; 12:457-472. [PubMed: 18805942]

23. Christensen L, Hutman T, Rozga A, Young GS, Ozonoff S, Rogers SJ, Baker B, Sigman M, et al. Play and developmental outcomes in infant siblings of children with autism. J Autism Dev Disord. 2010; 40:946-957. [PubMed: 20112084]

24. Loh A, Soman T, Brian J, et al. Stereotpyed motor behaviors associated with autism in high-risk infants: a pilot videotape analysis of a sibling sample. J Autism Dev Disord. 2007; 37:25-36. [PubMed: 17219059] 
25. Damiano CR, Nahmias A, Hogan-Brown AL, Stone WL. What do repetitive and stereotyped movements mean for infant siblings of children with autism spectrum disorders. J Autism Dev Disord. 2013; 43:1326-1335. [PubMed: 23080207]

26. Elison JT, Paterson SJ, Wolff JJ, et al. White matter microstructure and atypical visual orienting in 7- month-olds at risk for autism. Am J Psychiatry. 2013; 170:899-908. [PubMed: 23511344]

27. Wolff JJ, Gu H, Gerig G, et al. Differences in white matter fiber tract development present from 6 to 24 months in infants with autism. Am J Psychiatry. 2012; 169:589-600. [PubMed: 22362397]

28. Wetherby A, Allen L, Cleary J, Kublin K, Goldstein H. Validity and reliability of the communication and symbolic behavior scales developmental profile with very young children. $\mathrm{J}$ Speech Lang Hear Res. 2002; 45:1202-1218. [PubMed: 12546488]

29. Berument SK, Rutter M, Lord C, Pickles A, Bailey A. Autism screening questionnaire: diagnostic validity. Br J Psychiatry. 1999; 175:444-451. [PubMed: 10789276]

30. Lord C, Rutter M, Le Couteur A. Autism diagnostic interview-revised: a revised version of a diagnostic interview for caregivers of individuals with possible pervasive developmental disorders. J Autism Dev Disord. 1994; 24:659-685. [PubMed: 7814313]

31. Lord C, Risi S, Lambrecht L, et al. The autism diagnostic observation schedule-generic: a standardized measure of social and communication deficits associated with the spectrum of autism. J Autism Dev Disord. 2000; 30:205-223. [PubMed: 11055457]

32. Wetherby AM, Woods J, Allen L, Cleary J, Dickinson H, Lord C. Early indicators of autism spectrum disorders in the second year of life. J Autism Dev Disord. 2004; 34:473-493. [PubMed: 15628603]

33. Thelen E. Rhythmical stereotypies in normal human infants. Anim Behav. 1979; 27:699-715. [PubMed: 556122]

34. Mullen, EM. Mullen Scales of Early Learning: AGS Edition. Circle Pines, MN: AGS Publishing; 1995.

35. Kim SH, Lord C. Combining information from multiple sources for the diagnosis of autism spectrum disorders for toddlers and young preschoolers from 12 to 47 months of age. J Child Psychol Psychiatry. 2012; 53:143-151. [PubMed: 21883205]

36. Wolff JJ, Botteron KN, Dager SR, et al. Longitudinal patterns of repetitive behavior in toddlers with autism. J Child Psychol Psychiatry. 2014; 55:945-953. [PubMed: 24552513]

37. MacLean WE, Ellis DN, Galbreath HN, Halpern LF, Baumeister AA. Rhythmic motor behavior of perambulatory motor impaired, Down syndrome and nondisabled children: a comparative analysis. J Abnorm Child Psychol. 1991; 19:319-330. [PubMed: 1830892]

38. Iverson JM. Multimodality in infancy: vocal-motor and speech-gesture coordinations in typical and atypical development. Enfance. 2010; 3:257-274. [PubMed: 21494413]

39. Fenson L, Sapper V, Minner DG. Attention and manipulative play in the one-year-old child. Child Dev. 1974; 45:757-764. [PubMed: 4143830]

40. Oakes LM, Tellinghuisen DJ. Examining in infancy: does it reflect active processing? Dev Psychol. 1994; 30:748-756.

41. Ruff HA. Components of attention during infants' manipulative exploration. Child Dev. 1986; 57:105-114. [PubMed: 3948587]

42. Piaget, J. The Origin of Intelligence in Children. New York: International Universities Press, Inc; 1952.

43. Bishop SL, Richler J, Lord C. Association between restricted and repetitive behaviors and nonverbal IQ in children with autism spectrum disorders. Child Neuropsyc. 2006; 12:247-267.

44. Szatmari P, Maziade M, Zwaigenbaum L, et al. Informative phenotypes for genetic studies of psychiatric disorders. Am J Genet B Neuropsychiatr Genet. 2007; 144B:581-588.

45. Chawarska K, Klin A, Paul R, Macari S, Volkmar F. A prospective study of toddlers with ASD: short-term diagnostic and cognitive outcomes. J Child Psychol Psychiatry. 2009; 50:1235-1245. [PubMed: 19594835]

46. Guthrie W, Swineford LB, Nottke C, Wetherby AM. Early diagnosis of autism spectrum disorder: stability and change in clinical diagnosis and symptom presentation. J Child Psychol Psychiatry. 2013; 54:582-590. [PubMed: 23078094] 
47. Lord C. Follow-up of two-year-olds referred for possible autism. J Child Psychol Psychiatry. 1995; 36:1365-1382. [PubMed: 8988272]

48. Zwaigenbaum L, Bryson S, Lord C, et al. Clinical assessment and management of toddlers with suspected autism spectrum disorder: insights from studies of high-risk infants. Pediatrics. 2009; 123:1383-1391. [PubMed: 19403506]

49. Georgiades S, Szatmari P, Zwaigenbaum L, et al. A prospective study of autistic-like traits in unaffected siblings of probands with autism spectrum disorder. JAMA Psychiatry. 2013; 70:4248. [PubMed: 22945359]

50. Messinger D, Young GS, Ozonoff S, et al. Beyond autism: a baby sibling research consortium study of high-risk children at three years of age. J Am Acad Child Adolesc Psychiatry. 2013; 52:300-308. [PubMed: 23452686] 


\section{Clinical Guidance}

- An increased rate of stereotyped motor mannerisms may represent an early behavioral marker of ASD at 12 months of age.

- The manner by which 12-month-old high-risk siblings of children with ASD manipulate objects may not differentiate those who go on to develop ASD from those infants who do not.

- Careful consideration of motor development alongside social-communicative development in 12-month-olds may augment efforts aimed toward early identification of ASD. 


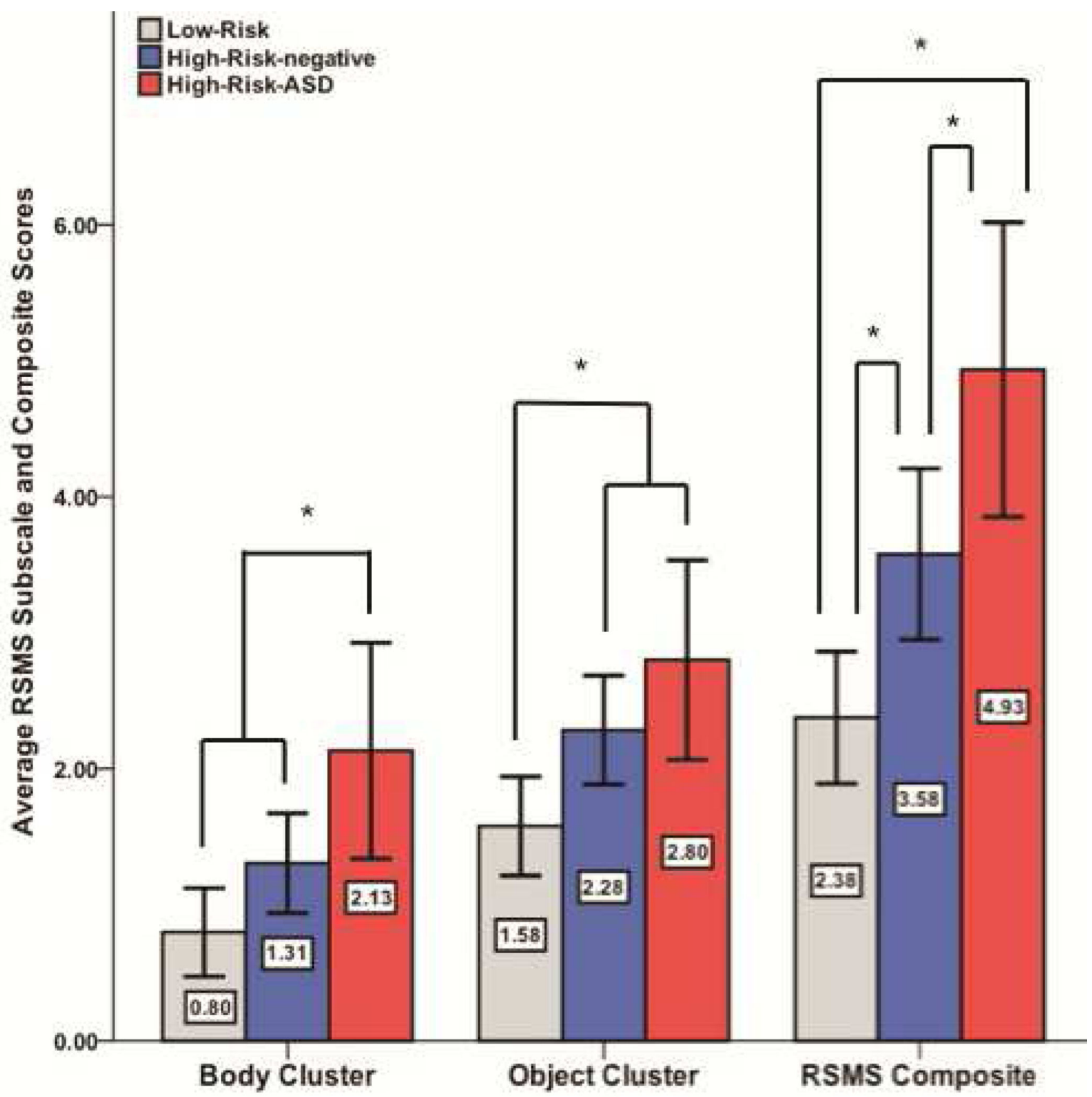

Figure 1.

Group differences in Repetitive and Stereotyped Movement Scales (RSMS) subscale and composite scores. Note: Error bars represent 2 standard errors; ASD = autism spectrum disorder. ${ }^{*} p<0.05$. 


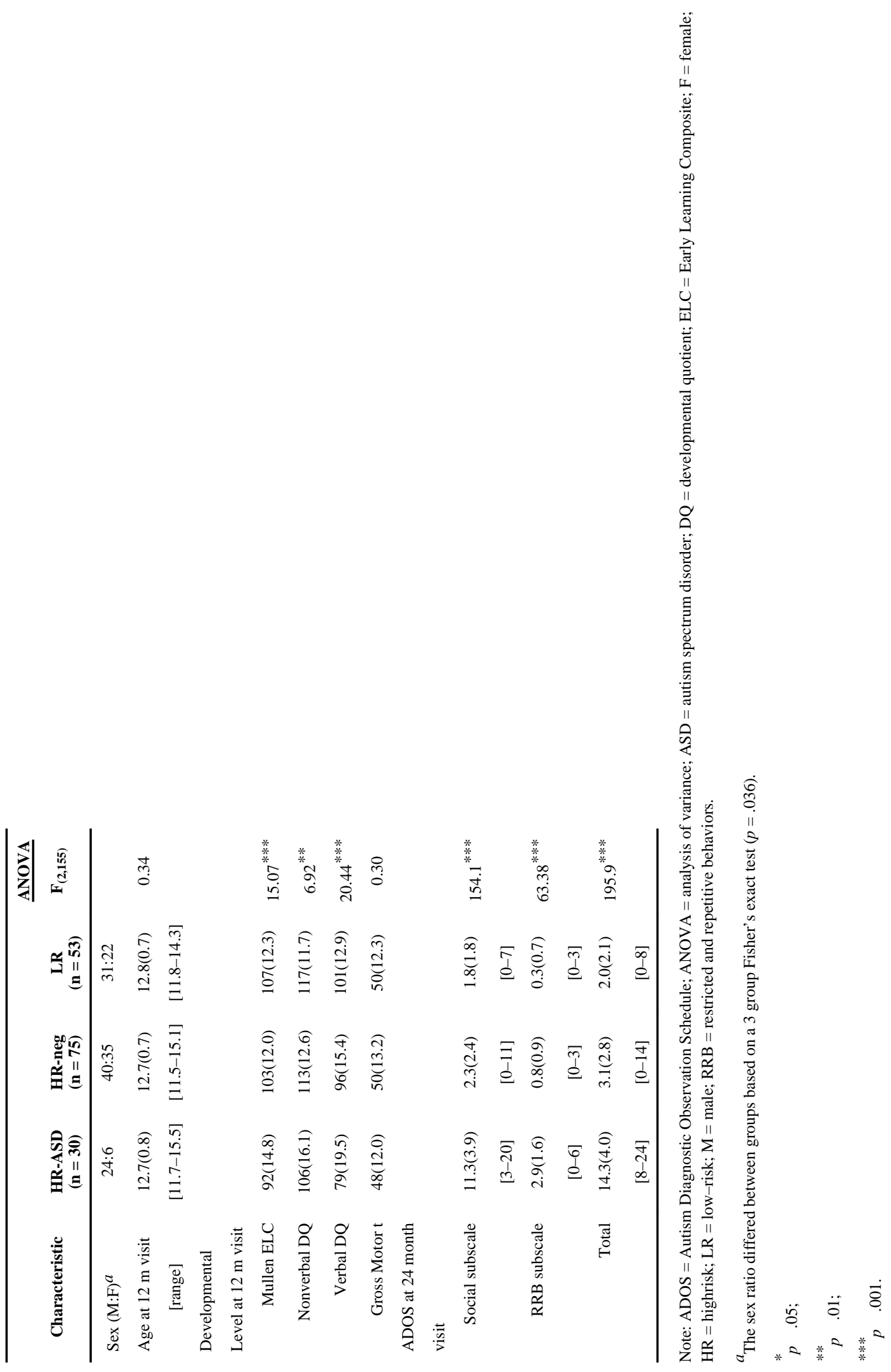




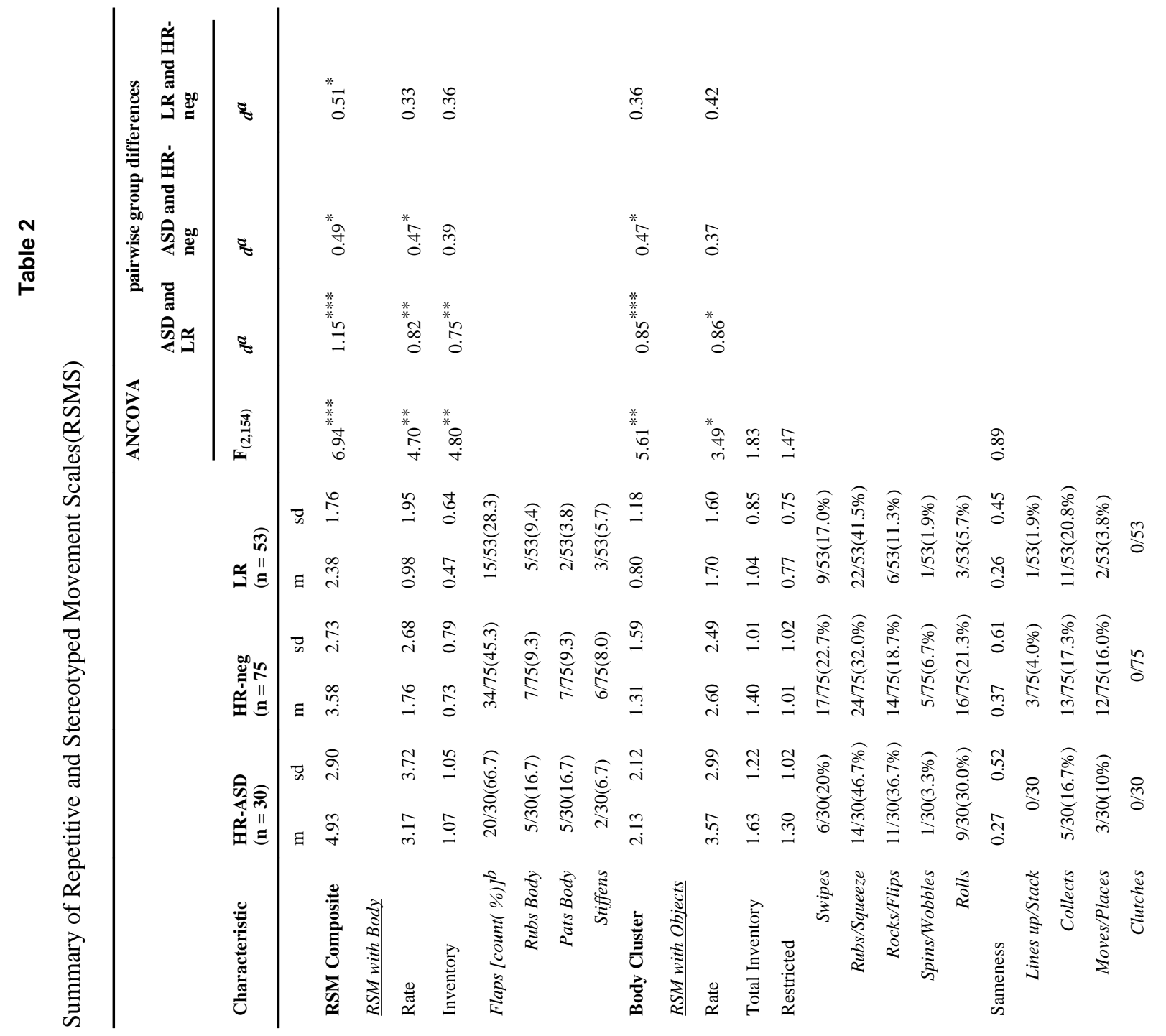


